ARTICLE

\title{
Substantial bulk photovoltaic effect enhancement via nanolayering
}

Fenggong Wang ${ }^{1}$, Steve M. Young ${ }^{2}$, Fan Zheng ${ }^{1}$, Ilya Grinberg ${ }^{1} \&$ Andrew M. Rappe ${ }^{1}$

Spontaneous polarization and inversion symmetry breaking in ferroelectric materials lead to their use as photovoltaic devices. However, further advancement of their applications are hindered by the paucity of ways of reducing bandgaps and enhancing photocurrent. By unravelling the correlation between ferroelectric materials' responses to solar irradiation and their local structure and electric polarization landscapes, here we show from first principles that substantial bulk photovoltaic effect enhancement can be achieved by nanolayering $\mathrm{PbTiO}_{3}$ with nickel ions and oxygen vacancies $\left(\left(\mathrm{PbNiO}_{2}\right)_{x}\left(\mathrm{PbTiO}_{3}\right)_{1-x}\right)$. The enhancement of the total photocurrent for different spacings between the $\mathrm{Ni}$-containing layers can be as high as 43 times due to a smaller bandgap and photocurrent direction alignment for all absorption energies. This is due to the electrostatic effect that arises from nanolayering. This opens up the possibility for control of the bulk photovoltaic effect in ferroelectric materials by nanoscale engineering of their structure and composition.

\footnotetext{
${ }^{1}$ The Makineni Theoretical Laboratories, Department of Chemistry, University of Pennsylvania, Philadelphia, Pennsylvania 19104-6323, USA. ${ }^{2}$ Center for Computational Materials Science, United States Naval Research Laboratory, Washington, DC 20375, USA. Correspondence and requests for materials should be addressed to F.W. (email: fenggong@sas.upenn.edu) or to A.M.R. (email: rappe@sas.upenn.edu).
} 
S tudies of photo-induced effects in ferroelectrics have experienced a revival due to the demonstration of a variety of fascinating physical effects, and in particular an increase in the power conversion efficiency of ferroelectric-based solar cells ${ }^{1-5}$. In particular, recent experiments on $\mathrm{BiFeO}_{3}$ thin films with absorption at the visible-light edge have revealed phenomena that were not observed in classic studies of bulk ferroelectric photoresponses ${ }^{6-9}$. Advances in the power conversion efficiency of ferroelectric-based solar cells and the discovery of visible-light-absorbing ferroelectric materials have provided further encouragement for this field ${ }^{3,4,10,11}$.

The recent experimental discoveries and progress in thin film fabrication have been accompanied by significant advances in the understanding of the unique light-matter interactions enabled by the presence of a spontaneous, switchable polarization in ferroelectric materials. In particular, it has been demonstrated that the very large open-circuit photovoltage observed in $\mathrm{BiFeO}_{3}$ films is due to the bulk photovoltaic effect (BPVE) ${ }^{12-15,7}$; in turn, BPVE has been shown to arise from the shift current mechanism, in which electrons are continuously excited under sustained illumination to quasiparticle coherent states that have intrinsic momenta, generating a spontaneous direct short-circuit photocurrent ${ }^{16,17}$. While the fundamental mechanism underlying BPVE has been determined, it is not yet completely understood how the BPVE depends on the structure of ferroelectric materials. In particular, a thorough understanding of how the BPVE can be controlled through adjustment of the atomic and electronic compositions and change of the chemical bonding properties is highly desired from the fundamental standpoint as well as for future development of BPVE-based devices.

Nano heterostructured materials have been shown to exhibit intriguing physics that are inaccessible in their bulk material counterparts due to the quantum confinement effect, the sensitivity of functional properties to structure, as well as the unique properties at interfaces, such as in the case of the two-dimensional electron gas at the $\mathrm{LaAlO}_{3} / \mathrm{SrTiO}_{3}$ interface ${ }^{18}$. Furthermore, it has been established that there is a connection between materials' local chemistry and structural properties, their fundamental and functional properties, and their responses to external stimuli ${ }^{19,20}$. Therefore, superior properties, specifically an enhancement of the photovoltaic performance, may be obtained by tuning the local structure and the landscape of local electric polarization in oxides with an engineered bandgap ${ }^{21}$. Motivated by the strong effect of electrostatics on materials' bandgaps and electronic transitions ${ }^{22,23}$, here we explore the possibility of nano-ordering photoresponsive ferroelectric materials to control and enhance the BPVE, highlighting the importance of length scale (layer thickness), electronics and electrostatics for photovoltaic performance.

We use first-principles calculations to investigate the relationship between local displacements, electronic structure and the BPVE response in the nanolayered $\mathrm{Ni}+$ vacancy-substituted $\mathrm{PbTiO}_{3}$ material $\mathrm{Pb}\left(\mathrm{Ni}_{x} \mathrm{Ti}_{1-x}\right) \mathrm{O}_{3-x}$ (called $\left(\mathrm{PbNiO}_{2}\right)_{x}$ $\left(\mathrm{PbTiO}_{3}\right)_{1-x}$ or PNT for brevity $)^{24,25}$. We choose Ni because it is a late-transition metal, and so it is relatively electronegative, with filled and empty $d$ orbitals near in energy; accordingly, $\mathrm{Ni}$ substitution induces a low-lying conduction band (CB). The combination of $\mathrm{Ni}$ and vacancy substitution guarantees that $\mathrm{Ni}$ ions are found in the +2 charge states. The $\mathrm{Ni}^{2+}$ state is also preferred to the $\mathrm{Ni}^{3+}$ state, because $\mathrm{Ni}^{3+}$ ion has a small ionic radius $(0.60 \AA)$ and is only stable in perovskites with small $\mathrm{La}^{3+}$ ions on the $A$ site, whereas the larger $\mathrm{Ni}^{2+}$ ion is known to be stable in ferroelectric compounds ${ }^{26}$. Because it is more computationally convenient, we choose PNT to serve as a prototype for the recently developed KBNNO material that for the first time showed excellent light absorption in the visible range by an oxide ferroelectric, breaking the decades-long restriction of ferroelectric oxides to only near-ultraviolet and ultraviolet absorption ${ }^{10}$. PNT is also closely related to the Nidoped $\mathrm{Pb}\left(\mathrm{Zr}_{1-x} \mathrm{Ti}_{x}\right) \mathrm{O}_{3-x}(\mathrm{PZT})$ material (PNZT) synthesized in thin film form and shown to exhibit improved light-absorption properties relative to the parent PZT material ${ }^{27}$. We show that the BPVE response over a wide range of frequencies is sensitive to the changes in the electronic structure that are driven by changes in the local cation displacements. The PNT nanostructures exhibit several unexpected and counterintuitive effects that enable the tuning of the absorption properties and a marked enhancement (by a factor of 43) of the BPVE response by nanoscale (1-2 nm) layering. Our results suggest that nanoscale control of composition and structure is an attractive route for manipulating the BPVE in ferroelectric oxides.

\section{Results}

Oxygen vacancy and cation arrangement. We start from exploring the relative stabilities of different $\mathrm{O}$ vacancy sites by choosing a $1 \times 1 \times 2$ supercell with one $\mathrm{Ti}$ atom substituted by $\mathrm{Ni}$ $\left(\mathrm{Ni}_{\mathrm{Ti}}\right)$ and one $\mathrm{O}$ atom removed $\left(V_{\mathrm{O}}\right)$, corresponding to the $\left(\mathrm{PbNiO}_{2}\right)\left(\mathrm{PbTiO}_{3}\right)$ composition $(x=1 / 2)$. Total energy analysis indicates that $\mathrm{O}$ vacancies prefer to form adjacent to $\mathrm{Ni}$ (Supplementary Fig. 1), because this provides more effective charge compensation. In addition, under normal conditions the equatorial $\mathrm{O}$ vacancies are more stable than the apical $\mathrm{O}$ vacancies. However, the apical $\mathrm{O}$ vacancies become more stable by $29 \mathrm{meV}$ per atom with $1 \%$ in-plane compressive strains. Furthermore, the apical O vacancies are preferred in pure $\mathrm{PbTiO}_{3}$ (ref. 28), and it is likely that a large proportion of the apical O vacancies are preserved after $\mathrm{Ni}$ incorporation.

The location of $\mathrm{O}$ vacancy can significantly affect the polarity of the structure and the composition of the contributing electronic states, and in turn may also affect the photocurrent. The shift current response of the $1 \times 1 \times 2$ supercell with equatorial $\mathrm{O}$ vacancies is almost negligible. This is because when equatorial $\mathrm{O}$ vacancies are introduced, the relaxed structure with $\mathrm{NiO}_{4}$ complexes in the vertical $y z$ (or $x z$ ) plane is nearly centrosymmetric, whereas the presence of apical $\mathrm{O}$ vacancies enlarges the lattice asymmetry. While emphasizing that a larger lattice asymmetry does not always induce a larger shift current, the obtained structural change is nevertheless consistent with the shift current results.

The $x=1 / 2$ composition may also take the rock salt $B$-cation arrangement (Supplementary Fig. 1), in which the $\mathrm{O}$ vacancy has no preferred site. Even though the rock salt cation arrangement is less energetically favourable than its layered counterpart, it exhibits a more substantial photocurrent response (Table 1). Comparison of their total and projected densities of states shows that the $\mathrm{Pb} 6 p$ orbitals contribute the most to the bottom of the $\mathrm{CB}$ in the rock salt structure, but in the layered structure the $\mathrm{Ni}$ $3 d$ orbitals contribute the most (Supplementary Fig. 1). This occurs because in the rock salt structure the $\mathrm{Ni}-V_{\mathrm{O}}-\mathrm{Ni}$ network is interrupted by the $\mathrm{Ti}$ and $\mathrm{Pb}$ atoms, making the $\mathrm{Ni} 3 d$ orbitals mainly localized at an energy position of $1 \mathrm{eV}$ higher than the bottom of the $\mathrm{CB}$. Electronic transitions involving the more delocalized $\mathrm{Pb} 6 p$ oribitals facilitate the motion of the photocurrent carriers and lead to stronger photocurrent response.

Photocurrent cancellation in PNT. Inspection of the shift current results (Fig. 1a; we focus only on the most substantial, $x x \mathrm{Z}$ component) for the $\mathrm{PbTiO}_{3}$ with $33 \% \mathrm{Ni}+$ vac composition $(1 \times 1 \times 3$ superlattice $)$ shows that the overall current produced by illumination in the visible range is small, due to a strong cancellation of the contributions for light absorption of photons 
Table 1 | Comparison of the photocurrent and electronic properties of different compositions of PNT.

\begin{tabular}{|c|c|c|c|c|c|c|}
\hline Structures & $\begin{array}{c}E_{\text {On }}^{\sigma} \\
(e V)\end{array}$ & $\left(10^{\left.\sigma_{\operatorname{Max}}^{-4} V^{-1}\right)}\right.$ & $\begin{array}{c}G_{\operatorname{Max}} \\
\left(10^{-9} \mathrm{~cm} \mathrm{~V}^{-1}\right)\end{array}$ & $\begin{array}{c}P \\
\left(C m^{-2}\right)\end{array}$ & $\begin{array}{l}E_{g}^{H S E} \\
(e V)\end{array}$ & $\begin{array}{c}\Delta E \\
m e V\end{array}$ \\
\hline $\mathrm{PbTiO}_{3}$ & 3.6 & 3.7 & 12.0 & 0.81 & 2.71 & - \\
\hline $1 \times 1 \times 2$ & 2.1 & 1.5 & 3.6 & 0.73 & 1.58 & 0 \\
\hline Rock salt & 2.1 & 6.1 & 11.0 & 0.96 & 2.05 & +30 \\
\hline $1 \times 1 \times 3$ & 1.9 & 3.9 & 14.0 & 0.91 & 1.58 & - \\
\hline $1 \times 1 \times 4$ & 1.6 & 4.7 & 29.7 & 1.10 & 1.33 & - \\
\hline $1 \times 1 \times 5$ & 1.5 & 4.5 & 36.9 & 1.07 & 1.28 & - \\
\hline $1 \times 1 \times 6$ & 1.5 & 6.7 & 46.8 & 1.05 & 1.08 & - \\
\hline
\end{tabular}

Calculated shift current onset energy $E_{\mathrm{O} n}^{\sigma}$, maximum shift current susceptibility $\sigma_{\mathrm{Max}}$, maximum Glass coefficient $G_{\text {Max }}$ polarization $P$ and Heyd-Scuseria-Ernzerhof (HSE) hybrid functional bandgap $E_{\mathrm{g}}^{\text {HSE }}$ of the layered and rock salt PNT. $\triangle E$ (meV per atom) is the relative stability of different cation arrangements that have the same composition. $\mathrm{PbTiO}_{3}$ values are also shown for comparison. Maximum values are for energies smaller than $3.0 \mathrm{eV}\left(E<4.0 \mathrm{eV}\right.$ for $\left.\mathrm{PbTiO}_{3}\right)$.

a
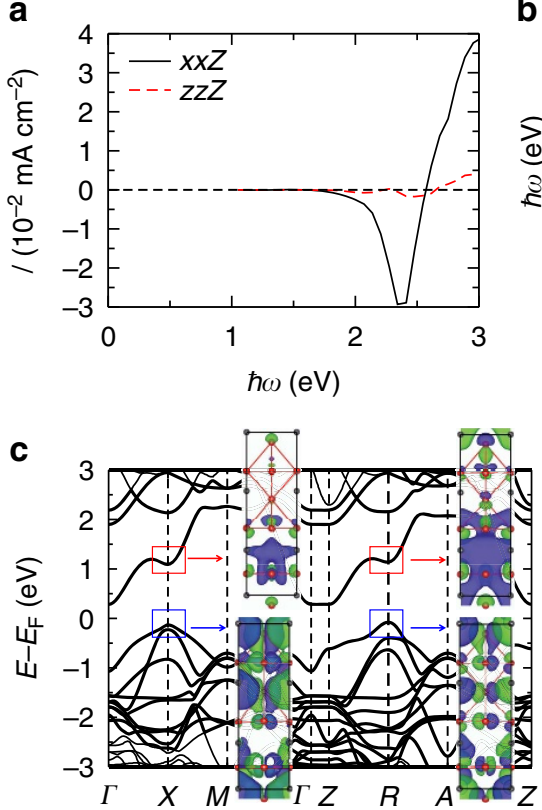

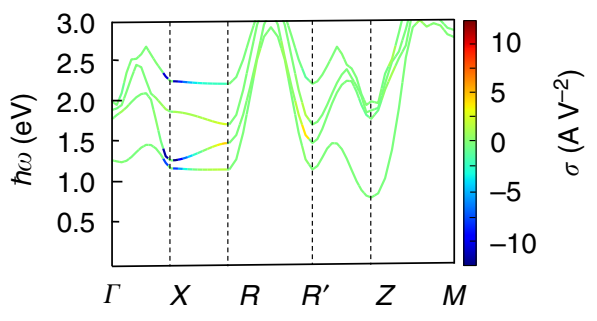

d

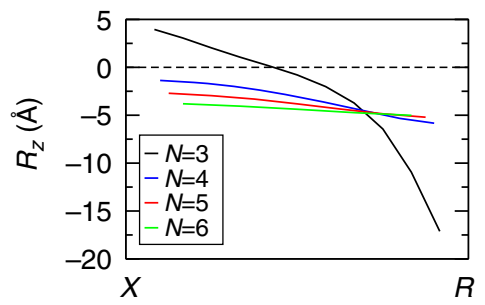

Figure 1 | The photocurrent cancellation in layered PNT and its electronic and wavefunction origins. The (a) photocurrent (assuming $0.1 \mathrm{~W} \mathrm{~cm}^{-2}$ light absorption), (b) $k$-resolved photocurrent and (c) band structure and real-space wavefunction isosurfaces corresponding to the states indicated by the rectangular regions, of the $1 \times 1 \times 3$-layered PNT. In $\mathbf{b}$, the colour gives the value of the photocurrent response $\sigma\left(\mathrm{AV}^{-2}\right)$. The near-gap response is dominated by the region around the line from $X$ to $R$, and changes direction along this path. $R^{\prime}$ indicates $R$ plus a reciprocal lattice vector, so that $R^{\prime}$ traverses the Brillouin zone. (d) The shift vector for the $k$ points along the $X-R$ line in the Brillouin zone of the $1 \times 1 \times N$-layered PNT.

in the $2-2.5 \mathrm{eV}$ range and in the $2.5-3.0 \mathrm{eV}$ range. Such a cancellation was also found by our theoretical calculations for the KBNNO material ${ }^{29}$. This is unlike the shift currents calculated for $\mathrm{PbTiO}_{3}$ and $\mathrm{BiFeO}_{3}$ that exhibit a uniform sign of the photoresponse throughout the photon spectrum. It is likely that the shift current cancellation observed in KBNNO and PNT is related to the greater diversity of electronic orbital character caused by the presence of dissimilar $\mathrm{Ni}$ and $\mathrm{Ti} / \mathrm{Nb}$ local environments. Examination of the $k$-resolved shift current (Fig. 1b) shows that the electronic transitions occurring near the $X(0,0.5,0)$ and $R(0,0.5,0.5) k$ points and along the $X-R$ line induce the most substantial photocurrent responses and are thus the most important for the band-edge electronic transitions. However, due to the changes in the direction of the shift vector (Fig. 1d), the contributions from the transitions at $X$ and at $R$ largely counteract each other. This suggests that the BPVE response can be significantly increased by aligning the sign of the photocurrent throughout the Brillouin zone.

To elucidate the origin of the photocurrent cancellation, we consider the details of the electronic states at the $X$ and $R$ points.
The calculated PNT band structure (Fig. 1c) shows that the bandgap is reduced due to the introduction by nickel of a low-lying $\mathrm{CB}$ into the bulk $\mathrm{PbTiO}_{3}$ gap; as a result, the band-edge electronic transitions, which will be responsible for the shift current response generated by the visible light, occur only between the valence bands and this particular CB. Real-space wavefunction distribution analysis for the highest valence band shows that it is essentially delocalized at both $X$ and $R$, with the wavefunction extending to the whole supercell because of the overlap among the $\mathrm{Pb} 6 s$, Ti $3 d, \mathrm{Ni} 3 d$ and $\mathrm{O} 2 p$ orbitals. However, the wavefunction distribution for the lowest $\mathrm{CB}$ shows substantial differences between $X$ and $R$. At $X$, it arises mainly from the Ni $3 d_{x^{2}-y^{2}}$ and the nearby $\mathrm{Pb} 6 p$ orbitals, and is more localized, whereas at $R$ it becomes much more delocalized and has larger orbital contributions from the $\mathrm{Pb} 6 p$, Ti $3 d_{x^{2}-y^{2}}$ and $\mathrm{Ti} 3 d_{z^{2}}$ orbitals. The greater delocalization at the $R$ point is directly related to the wavefunction character; compared with the Ni $3 d_{x^{2}-y^{2}}$ orbital, the $\mathrm{Pb} 6 p$ and $\mathrm{Ti} 3 d_{x^{2}-y^{2}}$ orbitals are more delocalized, while the $\mathrm{Ti}$ $3 d_{z^{2}}$ orbitals extend the wavefunction along the photocurrent direction. The increasing delocalization of the $\mathrm{CB}$ minimum 
a

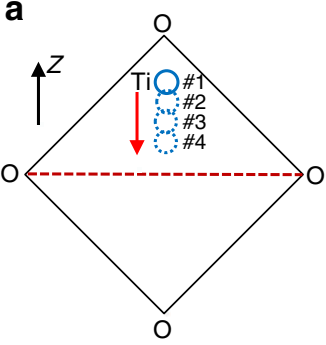

b

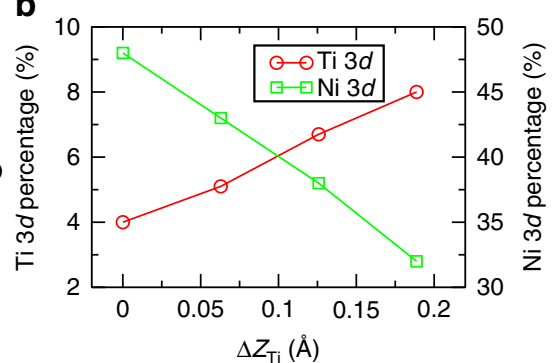

c

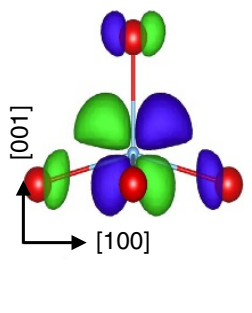

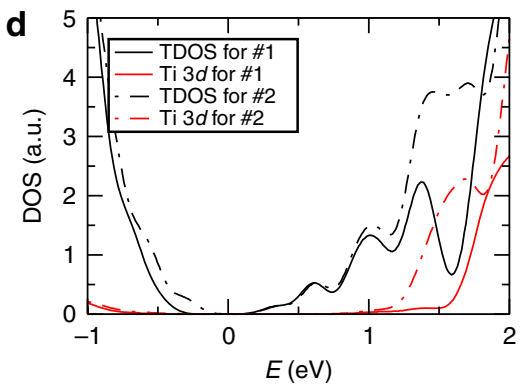

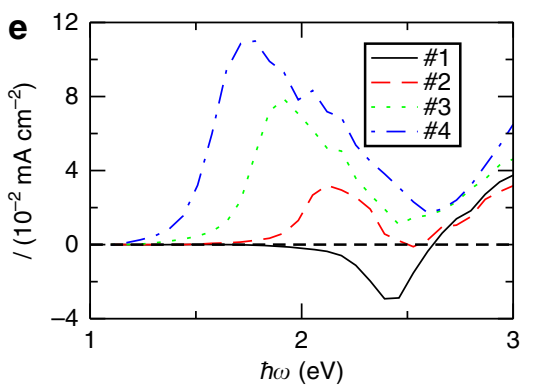

Figure 2 | The photocurrent enhancement by artificial local structure displacement and the corresponding change of the electronic composition. (a) The schematic representation of how the Ti atoms are moved with respect to the fully relaxed structure (structure \#1), (b) the percentages of the Ti and $\mathrm{Ni}$ $3 d$ orbitals at the $\mathrm{CB}\left(\sum_{m}\left|\left\langle\psi_{\mathrm{CB}, X} \mid \phi_{32 m}\right\rangle\right|^{2}\right)$ at $X k$ point as a function of the antiparallel displacement $\left(\Delta Z_{\mathrm{Ti}}\right)$ of Ti atoms, (c) the orbital character of the Ti-O orbital overlap at the CB, (d) total and projected density of states and (e) photocurrent (assuming for the 1,000 $\mathrm{Wm}^{-2}$ light absorption), of the $1 \times 1 \times 3$-layered PNT. The Ti atoms are moved antiparallel to the polarization, reducing but not entirely eliminating their off-centre displacements. The inset label \#n in a,d and $\mathbf{e}$ denotes the structures with different Ti antiparallel displacements. For example, \#1 denotes the fully relaxed structure and \#2, \#3 and \#4 denote the structures with $\mathrm{Ti}$ atoms displaced antiparallel by $0.06,0.12$ and $0.18 \AA$, respectively, with respect to their positions in \#1. Note that the Ti off-centre displacements in $\# 1$ are much larger than those in plain $\mathrm{PbTiO}_{3}$. In $\mathbf{b}$ and $\mathbf{c}$, when Ti moves towards the $\mathrm{O}_{4}$ plane, the Ti-O interaction becomes more of nonbonding and less of antibonding character, moving the Ti $3 d$ orbitals downward and enhancing their contribution to the band-edge electronic states. Also see Fig. $3 a$ for the Glass coefficient change under artificial Ti atom antiparallel displacement, and Supplementary Fig. 2 for the photocurrent change under artificial $\mathrm{Pb}$ atom displacement in the rock salt cation arrangement.

(CBM) wavefunction from $X$ to $R$ is accompanied by the change in the sign of the shift current and leads to a bigger shift vector magnitude (shift vector can be viewed as an indicator of the nonthermal carrier mobility) (Fig. 1d).

Photocurrent enhancement by artificial manipulation. The relationship between the CBM wavefunction character, delocalization and shift current sign suggests that eliminating or weakening the differences between the $X$ and $R$ point wavefunctions can largely eliminate the cancellation of counter-propagating currents and substantially increase the total BVPE response. To verify this, we engineer the CBM wavefunction by changing the chemical bonding in PNT. The bottom of the $\mathrm{CB}$ is composed of the $\mathrm{Ni} 3 d, \mathrm{Ti} 3 d, \mathrm{~Pb} 6 p$ and $\mathrm{O} 2 p$ orbitals, with the Ni $3 d$ orbitals making a major contribution (Supplementary Fig. 1). Since the peak of the Ti $3 d$ orbitals is located at a higher energy than that of the $\mathrm{Ni} 3 d$ peak, the contribution of the $\mathrm{Ti} 3 d$ states to the band-edge electronic transitions can be enhanced by a downshift of the Ti $3 d$ orbital energy at the CB (Fig. 2d). A reduced overlap between the Ti $3 d$ and $\mathrm{O} 2 p$ orbitals would give rise to a weaker Ti-O bond and a smaller energy level difference between the bonding (valence band) and antibonding (CB) $\mathrm{Ti}-\mathrm{O}$ orbitals, resulting in a downshift of the CBM Ti $3 d$ orbitals relative to the those of the relaxed structure ${ }^{30,31}$. Therefore, to shift the Ti $3 d$ states to lower energies, we increase the Ti-O distances in $1 \times 1 \times 3$ PNT by moving Ti atoms antiparallel to the overall polarization, reducing, but not entirely eliminating the local Ti off-centre displacements found in the relaxed structure (Fig. 2).
The resulting changes in the Ti-O bonding have three effects. First, analysis of the CBM density of states shows that the contribution of the $\mathrm{Ti} 3 d$ (and also $\mathrm{Pb} 6 p$, not shown) orbitals increases while the proportion of the $\mathrm{Ni} 3 d$ orbitals decreases steadily with $\mathrm{Ti}$ atomic movements (Fig. 2b), confirming that CBM electronic structure can be effectively controlled by changes in the local structure and Ti-O bonding. Second, the calculated bandgap decreases, leading to smaller photocurrent onset energy with greater artificial Ti sublattice displacement antiparallel to the polarization. Third, as the Ti sublattice is moved, this first leads to a change in the sign of the shift current direction, followed by a substantial enhancement of the shift current magnitude (Figs $2 \mathrm{e}$ and 3a). Following Planck's law of black-body radiation for solar power distribution over light frequency at $6,000 \mathrm{~K}$, the total photocurrent is enhanced by a factor of 97 , compared with the ground-state structure. This demonstrates that fairly gentle changes in the structure can significantly affect the CBM character and lead to a markedly enhanced BPVE response.

Photocurrent enhancement via nanolayering. The design principle of greater CBM delocalization elucidated by the study of artificial lattice adjustment can be realized in nanoscale heterostructures. They are essentially $\mathrm{PbNiO}_{2}$-doped $\mathrm{PbTiO}_{3}$, but can be viewed as a nanolayer heterostructure of $\mathrm{PbNiO}_{2}$ and $\mathrm{PbTiO}_{3}$. While a more rigorous temperature-dependent thermodynamic analysis is required for investigating its experimental viability, our $0 \mathrm{~K}$ density functional theory (DFT) stability analysis shows that these structures are very likely experimentally accessible in the $\mathrm{Pb}\left(\mathrm{Zr}_{0.2} \mathrm{Ti}_{0.8}\right)_{0.7} \mathrm{Ni}_{0.3} \mathrm{O}_{3-\delta}$ and 
KBNNO materials for which PNT serves as a prototype. We find that nanolayered structures are preferred to the rock salt-ordered PNT and that under $1 \%$ in-plane compressive strains, the $1 \times 1 \times 2$ superlattice with apical $\mathrm{O}$ vacancies is more stable than its equatorial counterpart. Thus, the enhancement of the photo-
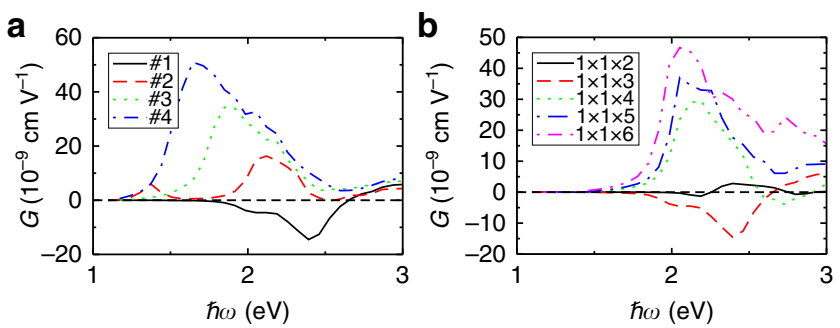

Figure 3 | The substantial enhancement of the Glass coefficient. The Glass coefficients of (a) the $1 \times 1 \times 3$ superlattice with different $\mathrm{Ti}$ antiparallel displacements and (b) the $1 \times 1 \times N$ superlattices. The inset label \#n in a denotes the structures with different Ti antiparallel displacements. For example, \#1 denotes the fully relaxed structure (no artificial antiparallel displacement) and \#2, \#3 and \#4 denote the structures with Ti atoms displaced antiparallel by $0.06,0.12$ and $0.18 \AA$, respectively, with respect to their positions in \#1. Note that the Ti off-centre displacements in $\# 1$ are much larger than those in plain $\mathrm{PbTiO}_{3}$. voltaic response found by our calculations can be realized through the growth of an epitaxially strained thin film or by layer deposition methods.

In PNT, the $\mathrm{Ni}_{\mathrm{Ti}}^{\prime \prime} \mathrm{O}_{2}$ layer has -2 charge, and is adjacent to the $\mathrm{Pb} V_{\mathrm{O}}$ layer with a +2 charge. Thus, $\mathrm{Ni}_{\mathrm{Ti}}^{\prime \prime}+V_{\mathrm{O}}$ substitution gives rise to charge separation. The Ni-vacancy layers also provide a polarizing field on the $\mathrm{PbTiO}_{3}$ layers, with this effect reduced as the $\mathrm{Ni}$-vacancy layers are spaced further apart. Approximating the layers as simple charged sheets, we obtain a periodic series of regions with electric field, analogous to the polar catastrophe in $\mathrm{LaAlO}_{3} / \mathrm{SrTiO}_{3}$ (ref. 18). As the separation between the $\mathrm{Ni}$ increases with increasing layer numbers $N$, the electric field in the bulk $\mathrm{PbTiO}_{3}$ layers becomes smaller under short-circuit boundary conditions, and bound charges screen the field over a greater length, delocalizing the electron densities (Fig. 4a). This mechanism for the control of wavefunction delocalization and photocurrent is confirmed by the analysis of the electronic structure and the calculated response for $1 \times 1 \times N(N=2-6)$ PNT supercells with one $\mathrm{NiO}_{2}$ plane per supercell. To show the changes due to the delocalization, we plot the layer-averaged probability density as a function of layer normal coordinate for the relevant $\mathrm{CBM}$ and valence band maximum (VBM) states (Fig. $4 \mathrm{~b}, \mathrm{c}$ ). Going from $N=3$ to 4 , these states, especially the valence band state, become more delocalized, corresponding to the enhancement of the shift current magnitude for a certain

a
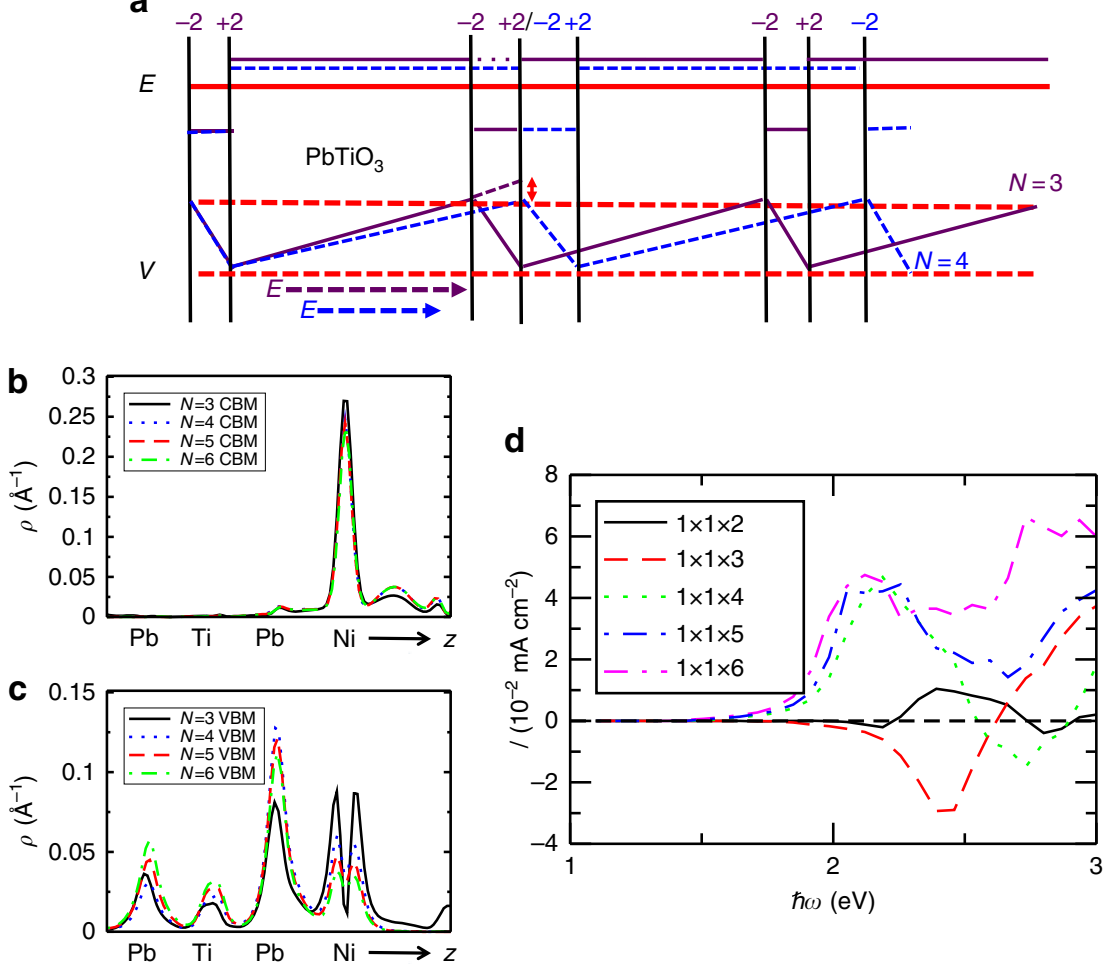

Figure 4 | The photocurrent enhancement via nanolayering and the corresponding change of the electric field landscape and state delocalization. (a) The electric field and potential inside PNT for $N=3$ and 4 . The $\mathrm{Ni}_{T_{i}}^{\prime \prime}+V_{0}$ substitution results in adjacent planes of charge. As $N$ increases, less screening over a greater distance is generated in response to the potential changes introduced by this charge separation. The electric field magnitude over the $\mathrm{PbTiO}_{3}$ layers is approximately $\frac{2 e}{(2 N-1) \varepsilon_{0}{ }^{\prime}}$ decreasing as $N$ increases. $(\mathbf{b}, \mathbf{c})$ The real-space probability density distribution summed over the Cartesian $x$ and $y$ coordinates $\left(\rho(z)=\int \mathrm{d} x \mathrm{~d} y\left|\left\langle\mathbf{r} \mid \psi_{n_{0}, R}\right\rangle\right|^{2}\right)$ for the CBM and valence band maximum (VBM) states at $R k$ point. $N$ is the number of layers. For $N>3$, the valence band wavefunction is localized within the supercell, yielding qualitatively distinct behaviour (as seen in Fig. 1c). The decreased electric field allows the wavefunction to decay more slowly, shifting the probability density of the valence band away from nickel, increasing the intracell delocalization. (d) The photocurrent (assuming $0.1 \mathrm{~W} \mathrm{~cm}^{-2}$ light absorption) of the layered PNT with varying superlattice periodicity. As $N$ increases, the near-gap response stabilizes and the response at $3 \mathrm{eV}$ increases; this represents an across-the-board increase in response per light-absorbing $\mathrm{Ni}$ layer due to greater delocalization. Increasing the superlattice $\mathrm{N}$ reduces the polarizing field on $\mathrm{PbTiO}_{3}$, aligning the shift current response across the visible range and increasing the current per absorbed photon. 
photon energy. In addition, this delocalization is only moderately enhanced from $N=4$ to 6 , resulting in the relatively flat progression of shift current at a particular photon energy observed for $N>3$.

Comparison of the calculated shift current spectra shows that contrary to naive expectations, reduction of the Ni fraction actually increases the effectiveness of substitution, increasing the response and reducing the bandgap as the number of layers is increased (Figs $3 \mathrm{~b}$ and $4 \mathrm{~d}$ ). This enhancement of the total photocurrent is due both to the enhancement of photocurrent magnitude at a certain photon energy and to the alignment of the photocurrent sign at different photon energies throughout the spectrum. The effect for visible-light illumination is quite marked, with an increase by a factor of 43 due to a simple insertion of additional bulk $\mathrm{PbTiO}_{3}$ layer(s). Such extraordinary sensitivity underscores the importance and usefulness of nanoscale ordering for the BPVE engineering. We find that the total shift current under visible-light illumination is also over 10 times greater than that for the $\mathrm{BiFeO}_{3}$ material that has been the standard for experiments on the photoresponse of ferroelectrics. The proximate reason for the enhancement in response becomes clear on looking at the response along the $X-R$ line (Fig. 1d and Supplementary Fig. 3). The difference between the $1 \times 1 \times 3$ and $1 \times 1 \times 4$ is due to the disappearance of the sign change versus $k$ point in the shift vector and the resulting shift current response. The shift current magnitude then further increases with the number of $\mathrm{PbTiO}_{3}$ layers. As $N$ increases further, the effect of eliminating cancellation of counter-propagating photocurrents at different photon energies starts to dominate, and this further enhances the total photocurrent for the whole visible-light spectrum.

\section{Discussion}

The nanoscale layered heterostructures have another potential benefit. Recent experimental work has highlighted the importance of conventional transport characteristics for BPVE ${ }^{7}$. Essentially, the performance of the bulk photovoltaic materials depends not only on their current generation capability but also on the photovoltage that they can sustain. This latter quantity is determined by the resistance of the current leaking back through the material, and the design of useful devices will therefore require control over conventional conductivity. In $\mathrm{BiFeO}_{3}$, domain walls can serve as barricades against such leakage, substantially increasing the resistance and, consequently, the maximum photovoltage. We note that the present system similarly features a nanoscale heterogeneous layered structure and may be viewed as a nanoscale composite with alternating photocurrent-generating layers and insulating layers, ideal for a BPVE device.

The foregoing holds important lessons for engineering bulk photovoltaic materials. First, it indicates that the problems of bandgap reduction and state delocalization can be partially separated. Nickel substitution provides lower-energy CB states that tend to be localized along the layer normal direction and are not favourable for a large shift current. Nevertheless, these states may participate in transitions with what are essentially $\mathrm{PbTiO}_{3}$ states that are delocalized and thus favour larger shift currents. We have chosen a material with robust shift current potential but a large gap $\left(\mathrm{PbTiO}_{3}\right)$, and introduced a modification that solves the latter without losing the former. Second, it emphasizes the importance of not only the magnitude of photocurrent as a factor in response, but consistency in direction, which is sensitive to relatively small structural changes, and therefore amenable to manipulation. Third, the electrostatic effects of the $\mathrm{Ni}_{\mathrm{Ti}}^{\prime \prime}+V_{\mathrm{O}}$ substitution on the electronic structure of bulk $\mathrm{PbTiO}_{3}$ are pronounced and most apparent for lower nickel fractions. We hope that our work will motivate further studies of photoresponse in nanoscale ferroelectrics.

The fact that a substantial enhancement of the BPVE is found in an ordered nanostructure highlights the importance of order/disorder effects in photoferroelectrics. It has been long accepted that most ferroelectrics and related materials exhibit local deviations from their average structures, whether in the form of disordered $B$-cation displacements in the conventional $\mathrm{BaTiO}_{3}$ and $\mathrm{KNbO}_{3}$ ferroelectrics, in relaxor ferroelectrics or in solid solutions ${ }^{20,32,33}$. The disorder in $\mathrm{BaTiO}_{3}$ and $\mathrm{KNbO}_{3}$ is due to the presence of ordered chains of displacements and that in the relaxor-like material $\mathrm{Na}_{1 / 2} \mathrm{Bi}_{1 / 2} \mathrm{TiO}_{3}$ is related to planar defects, and both of these structural features are found in our system $^{19,32}$. This order/disorder scenario leads to intriguing physics of electronic structure, phase transition, ferroelectricity, piezoelectricity, ferroelasticity, magnetism, and, in our case, the ferroelectric photovoltaic effect. Therefore, to obtain a good ferroelectric photovoltaic, attention should be paid to the effects of order/disorder and to the manipulation of the electric field landscape and bandgap via ordering/disordering; the present work serves as a good starting point for this approach.

Finally, we point out that our finding is not specific to the particular system studied here, but is rather general to a variety of ferroelectric oxide systems incorporating $\mathrm{Ni}$ (for example, $\mathrm{PbNiO}_{2}$-doped $\mathrm{PZT}$ and $\mathrm{KBNNO}$ ) and other $3 d$ transition metals. This is because the contributing electronic states in such a complex system are typically mixtures of different atomic orbitals that can be tailored by changes in structure and electrostatics, and by ordering/disordering. However, due both to the complexity of the relationship between photocurrent, electronic structure and atomic structure, and to the current paucity of visible-light-absorbing ferroelectric photovoltaic materials, a careful and detailed analysis will be required to estimate the magnitude of the nanolayering effects for each particular system.

\section{Methods}

First-principles calculation. A previously developed approach implemented in our in-house code that yields good agreement with experiment for shift current magnitude and spectral profile was used to calculate the shift current ${ }^{17,34}$. The QUANTUM-ESPRESSO code was used to perform DFT calculations with the local density approximation functional ${ }^{35-37}$. All elements are represented by normconserving, optimized nonlocal pseudopotentials ${ }^{38}$. For structural optimizations, Monkhorst-Pack grids ${ }^{39}$ with at least $6 \times 6 \times 6 k$-point meshes were used, while for the self-consistent and non-self-consistent calculations, finer $k$-point grids up to $40 \times 40 \times 40$ were used to get a well-converged shift current response. The DFT $+U$ method was used to improve the description of $d$-orbital electrons, with the Hubbard $U$ parameterized by the linear-response approach ${ }^{40}$. The calculated $U$ values are $\approx 4.7$ and $\approx 8.9 \mathrm{eV}$ for $\mathrm{Ti}$ and $\mathrm{Ni}$, respectively. All photon energies are shifted by $1.05 \mathrm{eV}$ so that $\mathrm{PbTiO}_{3}$ is at its experimental bandgap $(3.6 \mathrm{eV})$. Heyd-Scuseria-Ernzerhof hybrid functional calculations ${ }^{41}$ were also used to corroborate the bandgap trends obtained by DFT $+U$ calculations.

The shift current susceptibility tensor. Tetragonal $\mathrm{PbTiO}_{3}$ belongs to the $\mathrm{P} 4 \mathrm{~mm}$ space group, corresponding to the $C_{4 v}$ point group; in this case, the third rank shift current response tensor must have the form

$$
\sigma=\left[\begin{array}{cccccc}
0 & 0 & 0 & 0 & \sigma_{z y Y} & 0 \\
0 & 0 & 0 & \sigma_{z y Y} & 0 & 0 \\
\sigma_{x x Z} & \sigma_{x x Z} & \sigma_{z z Z} & 0 & 0 & 0
\end{array}\right]
$$

where the lower- and upper-case letters represent directions of the light polarization and photocurrent, respectively. The Glass coefficient

$$
G_{r r q}=\frac{\sigma_{r r q}(\omega)}{\alpha_{r r}(\omega)}
$$

describes the current response in a thick sample and includes the light attenuation effect due to the absorption coefficient $\alpha_{r r}(\omega)$. We choose our polarization direction to be normal to the Ni layers, so that all analysed systems have the same symmetry properties, and (for unpolarized light) only the $x x Z$ and $z z Z$ components are relevant. In PNT, the shift current induced by the perpendicularly polarized light $(x x Z)$ is much stronger than that induced by the parallel polarized light $(z z Z)$ 
because of their different absorption efficiencies. In the present work, we focus only on the more substantial $x x Z$ component.

\section{References}

1. Choi, T., Lee, S., Choi, Y. J., Kiryukhin, V. \& Cheong, S.-W. Switchable ferroelectric diode and photovoltaic effect in $\mathrm{BiFeO}_{3}$. Science 324, 63-66 (2009).

2. Kundys, B., Viret, M., Colson, D. \& Kundys, D. O. Light-induced size changes in $\mathrm{BiFeO}_{3}$ crystals. Nat. Mater. 9, 803-805 (2010).

3. Qin, M., Yao, K. \& Liang, Y. C. High efficient photovoltaics in nanoscaled ferroelectric thin films. Appl. Phys. Lett. 93, 122904 (2008).

4. Cao, D. et al. High-efficiency ferroelectric-film solar cells with an n-type $\mathrm{Cu}_{2} \mathrm{O}$ cathode buffer layer. Nano Lett. 12, 2803-2809 (2012).

5. Alexe, M. \& Hesse, D. Tip-enhanced photovoltaic effects in bismuth ferrite. Nat. Commun. 2, 256 (2011).

6. Fridkin, V. M. Bulk photovoltaic effect in noncentrosymmetric crystals. Crystallogr. Rep. 46, 654-658 (2001).

7. Bhatnagar, A., Chaudhuri, A. R., Kim, Y. H., Hesse, D. \& Alexe, M. Role of domain walls in the abnormal photovoltaic effect in $\mathrm{BiFeO}_{3}$. Nat. Commun. 4, 2835 (2013).

8. Yang, S. Y. et al. Above-bandgap voltages from ferroelectric photovoltaic devices. Nat. Nanotechnol. 5, 143 (2010).

9. Yang, S. Y. et al. Photovoltaic effects in $\mathrm{BiFeO}_{3}$. Appl. Phys. Lett. 95, 062909 (2009).

10. Grinberg, I. et al. Perovskite oxides for visible-light-absorbing ferroelectric and photovoltaic materials. Nature 503, 509-512 (2013).

11. Nechache, R. et al. Bandgap tuning of multiferroic oxide solar cells. Nat. Photon. 9, 61-67 (2015).

12. Glass, A. M., von der Linde, D. \& Negran, T. J. High-voltage bulk photovoltaic effect and the photorefractive process in $\mathrm{LiNbO}_{3}$. Appl. Phys. Lett. 1925, 233-235 (1974)

13. Kraut, W. \& von Baltz, R. Anomalous bulk photovoltaic effect in ferroelectrics: a quadratic response theory. Phys. Rev. B 19, 1548-1554 (1979).

14. Chynoweth, A. G. Surface space-charge layers in barium titanate. Phys. Rev. 102, 705-714 (1956).

15. Ji, W., Yao, K. \& Liang, Y. C. Bulk photovoltaic effect at visible wavelength in epitaxial ferroelectric $\mathrm{BiFeO}_{3}$ thin films. Adv. Mater. 22, 1763-1766 (2010).

16. von Baltz, R. \& Kraut, W. Theory of the bulk photovoltaic effect in pure crystals. Phys. Rev. B 23, 5590-5596 (1981).

17. Young, S. M. \& Rappe, A. M. First principles calculation of the shift current photovoltaic effect in ferroelectrics. Phys. Rev. Lett. 109, 116601 (2012).

18. Chtomo, A. \& Hwang, H. Y. A high-mobility electron gas at the $\mathrm{LaAlO}_{3} / \mathrm{SrTiO}_{3}$ heterointerface. Nature 427, 423-426 (2004).

19. Kreisel, J. et al. High-pressure x-ray scattering of oxides with a nanoscale local structure: Applications to $\mathrm{Na}_{1 / 2} \mathrm{Bi}_{1 / 2} \mathrm{TiO}_{3}$. Phys. Rev. B 68, 014113 (2003).

20. Grinberg, I., Cooper, V. R. \& Rappe, A. M. Relationship between local structure and phase transitions of a disordered solid solution. Nature 419, 909 (2002).

21. Kreisel, J., Alexe, M. \& Thomas, P. A. A photoferroelectric material is more than the sum of its parts. Nat. Mater. 11, 260 (2012).

22. Balachandran, P. V. \& Rondinelli, J. M. Massive band gap variation in layered oxides through cation ordering. Nat. Commun. 6, 6191 (2015)

23. Liu, S. et al. Ferroelectric domain wall induced band gap reduction and charge separation in organometal halide perovskites. J. Phys. Chem. Lett. 6, 693-699 (2015).

24. Bennett, J. W., Grinberg, I. \& Rappe, A. M. New highly polar semiconductor fer-roelectrics through $d^{8}$ cation-O vacancy substitution into $\mathrm{PbTiO}_{3}$ : a theoretical study. J. Am. Chem. Soc. 130, 17409-17412 (2008).

25. Gou, G. Y., Bennett, J. W., Takenaka, H. \& Rappe, A. M. Post density functional theoretical studies of highly polar semiconductor $\mathrm{Pb}\left(\mathrm{Ti}_{1-\mathrm{x}} \mathrm{Ni}_{\mathrm{x}}\right) \mathrm{O}_{3-\mathrm{x}}$ solid solutions: effects of cation arrangement on band gap. Phys. Rev. B 83, 205115 (2011).

26. Kondo, M. \& Kurihara, K. Sintering behavior and surface microstructure of $\mathrm{PbO}$-rich $\mathrm{PbNi}_{1 / 3} \mathrm{Nb}_{2 / 3} \mathrm{O}_{3}-\mathrm{PbTiO}_{3}-\mathrm{PbZrO}_{3}$ ceramics. J. Am. Ceram. Soc. 84, 2469-2474 (2001)

27. Kumari, S., Crtega, N., Kumar, A., Scott, J. F. \& Katiyar, R. S. Ferroelectric and photovoltaic properties of transitional metal doped $\mathrm{Pb}\left(\mathrm{Zr}_{0.14} \mathrm{Ti}_{0.56} \mathrm{Ni}_{0.30}\right) \mathrm{O}_{3-\delta}$ thin films. AIP Adv. 4, 037101 (2014).

28. Zhang, Z., Wu, P., Lu, L. \& Shu, C. Study on vacancy formation in ferroelectric $\mathrm{PbTiO}_{3}$ from ab initio. Appl. Phys. Lett. 88, 142902 (2006).
29. Wang, F. \& Rappe, A. M. First-principles calculation of the bulk photovoltaic effect in $\mathrm{KNbO}_{3}$ and $(\mathrm{K}, \mathrm{Ba})(\mathrm{Ni}, \mathrm{Nb}) \mathrm{O}_{3}$. . Phys. Rev. B 91, 165124 (2015).

30. Wang, F., Grinberg, I. \& Rappe, A. M. Band gap engineering strategy via polarization rotation in perovskite ferroelectrics. Appl. Phys. Lett. 104, 152903 (2014).

31. Eng, H. W., Barnes, P. W., Auer, B. M. \& Woodward, P. M. Investigation of the electronic structure of $d^{0}$ transition metal oxides belonging to the perovskite family. J. Solid State Chem. 175, 94-109 (2003).

32. Comes, R., Lambert \& Guinier, A. The chain structure of $\mathrm{BaTiO}_{3}$ and $\mathrm{KNbO}_{3}$. Solid State Commun. 6, 715 (1968)

33. Burns, G. \& Dacol, F. H. Crystalline ferroelectrics with glassy polarization behavior. Phys. Rev. B 28, 2527 (1983).

34. Young, S. M., Zheng, F. \& Rappe, A. M. First-principles calculation of the bulk photovoltaic effect in bismuth ferrite. Phys. Rev. Lett. 109, 236601 (2012).

35. Giannozzi, P. et al. Quantum Espresso: a modular and open-source software project for quantum simulations of materials. J. Phys. Condens. Matter 21, 395502 (2009).

36. Kohn, W. \& Sham, L. J. Self-consistent equations including exchange and correlation effects. Phys. Rev. 140, A1133-A1138 (1965).

37. Perdew, J. P. \& Zunger, A. Self-interaction correction to density-functional approximations for many-electron systems. Phys. Rev. B 23, 5048-5079 (1981).

38. Rappe, A. M., Rabe, K. M., Kaxiras, E. \& Joannopoulos, J. D. Optimized pseudopotentials. Phys. Rev. B 41, 1227-1230 (1990).

39. Monkhorst, H. J. \& Pack, J. D. Special points for Brillouin-zone integrations. Phys. Rev. B 13, 5188-5192 (1976)

40. Cococcioni, M. \& de Gironcoli, S. Linear response approach to the calculation of the effective interaction parameters in the LDA $+U$ approach. Phys. Rev. $B$ 71, 035105 (2005)

41. Heyd, J., Scuseria, G. E. \& Ernzerhof, M. Hybrid functionals based on a screened Coulomb potential. J. Chem. Phys. 118, 8207-8215 (2003).

\section{Acknowledgements}

F.W. was supported by the National Science Foundation, under Grant DMR-1124696. S.M.Y. was supported by the Department of Energy Office of Basic Energy Sciences under Grant DE-FG02-07ER46431, and a National Research Council Research Associateship Award at the US Naval Research Laboratory. F.Z. was supported by the National Science Foundation under Grant CMMI-1334241. I.G. was supported by the Office of Naval Research under Grant N00014-12-1-1033. A.M.R. was supported by the Office of Naval Research under Grant N00014-11-1-0664. Computational support was provided by the High-Performance Computing Modernization Office of the Department of Defense and the National Energy Research Scientific Computing Center of the Department of Energy.

\section{Author contributions}

F.W. performed the calculations; F.W. and S.M.Y. performed the data processing and analysis; F.W., S.M.Y. and I.G. wrote the manuscript; A.M.R. supervised the work; all authors contributed to the conception of ideas, to discussions, and to manuscript modification.

\section{Additional information}

Supplementary Information accompanies this paper at http://www.nature.com/ naturecommunications

Competing financial interests: The authors declare no competing financial interests

Reprints and permission information is available online at http://npg.nature.com/ reprintsandpermissions/

How to cite this article: Wang, F. et al. Substantial bulk photovoltaic effect enhancemen via nanolayering. Nat. Commun. 7:10419 doi: 10.1038/ncomms10419 (2016).

This work is licensed under a Creative Commons Attribution 4.0 International License. The images or other third party material in this article are included in the article's Creative Commons license, unless indicated otherwise in the credit line; if the material is not included under the Creative Commons license, users will need to obtain permission from the license holder to reproduce the material To view a copy of this license, visit http://creativecommons.org/licenses/by/4.0/ 